\title{
Study of the Motion of a Weighted Cotton Ball in an Air Stream with a Known Impact Trajectory
}

\author{
Mamatqulov Orifjon Tursunovich*, Juraeva Gulkhayo Ravshanbekovna, \\ Kambarov Elmurod Akhmadali Ugli \\ Technological Machine and Equipments, Namangan Institute of Engineering and Technology, Namangan, Uzbekistan \\ Email: ^m.orif@mail.ru, juraeva89@list.ru, elmurodqambarow@mail.ru
}

How to cite this paper: Tursunovich, M.O., Ravshanbekovna, J.G. and Ugli, K.E.A. (2020) Study of the Motion of a Weighted Cotton Ball in an Air Stream with a Known Impact Trajectory. Engineering, 12, 886-892. https://doi.org/10.4236/eng.2020.1212062

Received: October 19, 2020

Accepted: December 25, 2020

Published: December 28, 2020

Copyright $\odot 2020$ by author(s) and Scientific Research Publishing Inc. This work is licensed under the Creative Commons Attribution International License (CC BY 4.0).

http://creativecommons.org/licenses/by/4.0/

\begin{abstract}
In the cotton factories ginning process bales of raw cotton in cotton tube transporting through the air has written in the article. As a result, cotton with cotton in the air separated by a separator device with air traffic, has an important theoretical study. First of all, the air flow that affects the dynamic pressure and laws is based on the model established in this study and the results are obtained.
\end{abstract}

\section{Keywords}

Cotton, Air, Fiber, Friction, Motion, Working Camera, Pressure, Transportation, Additions, Speed

\section{Introduction}

It is known that cotton raw is transported through the air with tube to the process of cotton ginning from bales. The forces of air flow, which is interaction with the properties of their bodies, were studied comprehensively and thoroughly in the hydrodynamic science. Depending on the flow velocity, aerodynamic force is formed in the body with influence of the flow. If the flow rate is not high, usually, the value of this power will be proportional to the speed of an air and object. That's why, it's important to study the movement of cotton with air theoretically [1] [2].

\section{Creating the Mathematical Models and Differential Equations of Motion of the Impact of the Air Flow of the Pieces of Cotton}

Let's imagine, impact of the air flow object, in two-dimensional system $x 0 y$ trajectory equation $y=y(x)$ moves the curves $B C$ (Figure 1 ). Flow velocity vec- 
tor is determined with $\boldsymbol{V}_{0}=\left\{V_{0 x}, V_{0 y}\right\}$ and other velocity vector is determined with $\boldsymbol{V}=\left\{V_{x}, V_{y}\right\}$. If the vector of the pieces is determined by the velocity vector. If the vector $V_{0}$ instruction from the angle of the set $\alpha_{0}$, and then use the official 2.1. [3].

$$
V_{0 x}=\left|V_{0}\right| \cos \alpha_{0}, \quad V_{0 y}=\left|V_{0}\right| \sin \alpha_{0}
$$

Equations are made. Air resistance force vectors $\boldsymbol{F}\left(V_{0 x}-V_{x}, V_{0 y}-V_{y}\right)$ according to the law of aerodinamics, relative force vector is directed to the vector. $\boldsymbol{V}_{0}-\boldsymbol{V}$ If this power's module

$$
\left|\boldsymbol{F}\left(V_{0 x}-V_{x}, V_{0 y}-V_{y}\right)\right|
$$

the relative speed module is marked:

$$
\left|\boldsymbol{V}_{0}-\boldsymbol{V}\right|=\sqrt{\left(V_{0 x}-V_{x}\right)^{2}+\left(V_{0 y}-V_{y}\right)^{2}}
$$

then the effect on the strength of the flow of air will be able to write the following vector $\boldsymbol{F}\left(V_{0 x}-V_{x}, V_{0 y}-V_{y}\right)=\mid \boldsymbol{F}\left(V_{0 x}-V_{x}, V_{0 y}-V_{y}\right)\left[\frac{V_{0 x}-V_{x}}{\left|\boldsymbol{V}_{0}-\boldsymbol{V}\right|} \boldsymbol{i}+\frac{V_{0 y}-V_{y}}{\left|\boldsymbol{V}_{0}-\boldsymbol{V}\right|} \boldsymbol{j}\right]$

Here $(\boldsymbol{i}, \boldsymbol{j})$ is a unit vector which directed to $0 x$ and $0 y$ arrow. The power and influence of the air flow $0 x$ and $0 y$ arrows projections expressed through the following formula [4]

$$
\begin{aligned}
& F_{x}=\left|\boldsymbol{F}\left(V_{0 x}-V_{x}, V_{0 y}-V_{y}\right)\right| \frac{V_{0 x}-V_{x}}{\left|\boldsymbol{V}_{0}-\boldsymbol{V}\right|}, \\
& F_{y}=\left|\boldsymbol{F}\left(V_{0 x}-V_{x}, V_{0 y}-V_{y}\right)\right| \frac{V_{0 y}-V_{y}}{\left|\boldsymbol{V}_{0}-\boldsymbol{V}\right|}
\end{aligned}
$$

Let's imagine, air resistance is proportional to the relative speed, the force vector and the relative speed law, the following will be

$$
\boldsymbol{F}\left(V_{0 x}-V_{x}, V_{0 y}-V_{y}\right)=C_{0}\left(\boldsymbol{V}_{0}-\boldsymbol{V}\right)
$$

here $C_{0}$-is coefficient determined by the experience.

The power module of this formula:

$$
\left|\boldsymbol{F}\left(V_{0 x}-V_{x}, V_{0 y}-V_{y}\right)\right|=C_{0} \sqrt{\left(V_{0 x}-V_{x}\right)^{2}+\left(V_{0 y}-V_{y}\right)^{2}}
$$

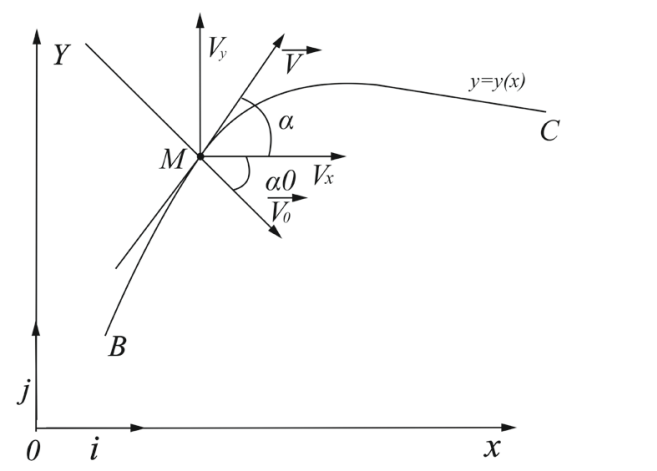

Figure 1. Scheme of the influence of the movement of air flow of the cotton pieces. 
It's projection of coordinate arrow will be:

$$
\begin{gathered}
F_{x}=C_{0} \sqrt{\left(V_{0 x}-V_{x}\right)^{2}+\left(V_{0 y}-V_{y}\right)^{2}} \frac{V_{0 x}-V_{x}}{\left|\boldsymbol{V}_{0}-\boldsymbol{V}\right|}=C_{0}\left(V_{0 x}-V_{x}\right) \\
F_{y}=C_{0} \sqrt{\left(V_{0 x}-V_{x}\right)^{2}+\left(V_{0 y}-V_{y}\right)^{2}} \frac{V_{0 y}-V_{y}}{\left|\boldsymbol{V}_{0}-\boldsymbol{V}\right|}=C_{0}\left(V_{0 y}-V_{y}\right)
\end{gathered}
$$

(2.4) and (2.5a) formulas air flow rate can be used as a small practice.

Now let's assume that is proportional to the square of the speed of the relative strength of the air flow,

$$
\boldsymbol{F}\left(V_{0 x}-V_{x}, V_{0 y}-V_{y}\right)=C_{1}\left(\boldsymbol{V}_{0}-\boldsymbol{V}\right)^{2}
$$

here $C_{1}$-coefficient which can be find by experience.

We find by using the properties of the vector squared module,

$$
\boldsymbol{F}\left(V_{0 x}, V_{0 y}-V_{y}\right)=C_{1}\left[\left(V_{0 x}-V_{x}\right)^{2}+\left(V_{0 y}-V_{y}\right)^{2}\right]
$$

in this case, look at the following:

$$
\begin{aligned}
F_{x} & =C_{1}\left[\left(V_{0 x}-V_{x}\right)^{2}+\left(V_{0 y}-V_{y}\right)^{2}\right] \frac{V_{0 x}-V_{x}}{\sqrt{\left(V_{0 x}-V_{x}\right)^{2}+\left(V_{0 y}-V_{y}\right)^{2}}} \\
& =C_{1}\left(V_{0 x}-V_{x}\right) \sqrt{\left(V_{0 x}-V_{x}\right)^{2}+\left(V_{0 y}-V_{y}\right)^{2}} \\
F_{y} & =C_{1}\left[\left(V_{0 x}-V_{x}\right)^{2}+\left(V_{0 y}-V_{y}\right)^{2}\right] \frac{V_{0 y}-V_{y}}{\sqrt{\left(V_{0 x}-V_{x}\right)^{2}+\left(V_{0 y}-V_{y}\right)^{2}}} \\
& =C_{1}\left(V_{0 y}-V_{y}\right) \sqrt{\left(V_{0 x}-V_{x}\right)^{2}+\left(V_{0 y}-V_{y}\right)^{2}}
\end{aligned}
$$

(2.5b) and (2.6) will be suitable for high-speed streams of the formula.

Now consider the following question. Suppose torque equilibrium position of the slice of cotton $t=0$, may affect the air flow equation slice trajectory $y=f(x)$. Examine these pieces of law. Let it play an angle $\gamma=f^{\prime}(x)$ with the axis vector of the flow of air $0 x$. Power is proportional to the relative speed of the air flow. In that case, the flow of air forces and missiles will be the following projections of $0 x$ and $0 y$ axis

$$
\begin{aligned}
& F_{x}=C_{0} \sqrt{\left(V_{0 x}-V_{x}\right)^{2}+\left(V_{0 y}-V_{y}\right)^{2}} \frac{V_{0 x}-V_{x}}{\left|\boldsymbol{V}_{0}-\boldsymbol{V}\right|}=C_{0}\left(V_{0} \cos \gamma-V_{x}\right) \\
& F_{y}=C_{0} \sqrt{\left(V_{0 x}-V_{x}\right)^{2}+\left(V_{0 y}-V_{y}\right)^{2}} \frac{V_{0 y}-V_{y}}{\left|\boldsymbol{V}_{0}-\boldsymbol{V}\right|}=C_{0}\left(V_{0} \sin \gamma-V_{y}\right)
\end{aligned}
$$

Here: $\cos \gamma=\frac{1}{\sqrt{1+f^{\prime 2}(x)}}, \sin \gamma=\frac{f^{\prime}(x)}{\sqrt{1+f^{\prime 2}(x)}}$.

Issue 1:

The mass $m$ of the pieces of cotton the weight of the air flow and its effect on the progress we make on the basis of the principle of equation Dalamber,

$$
m \ddot{x}=m \dot{V}_{x}=F_{x}=C_{0}\left(V_{0} \cos \gamma-V_{x}\right)=\frac{C_{0}}{\sqrt{1+f^{\prime 2}}}\left[V_{0}-V_{x} \sqrt{1+f^{\prime 2}}\right]
$$




$$
\begin{aligned}
m \ddot{y} & =m \dot{V}_{y}=F_{y}-m g=C_{0}\left(V_{0} \sin \gamma-V_{y}\right) \\
& =\frac{C_{0}}{\sqrt{1+f^{\prime 2}}}\left[V_{0} f^{\prime}-V_{y} \sqrt{1+f^{\prime 2}}\right]-m g
\end{aligned}
$$

In particular, if $y=k x+b$ (air flow directed along the line of effecting correct), then $f^{\prime}=k,(2.8)$ and (2.9) will look at the following equations $\left(\gamma_{0}=\operatorname{arctg} k\right)$ :

$$
\begin{aligned}
& m \ddot{x}=m \dot{V}_{x}=C_{0}\left(V_{0} \cos \gamma_{0}-V_{x}\right) \\
& m \ddot{y}=m \dot{V}_{y}=C_{0}\left(V_{0} \sin \gamma_{0}-V_{y}\right)
\end{aligned}
$$

These equations $x(0)=x_{0}, y(0)=y_{0}, \dot{x}(0)=v_{0 x}, \dot{y}(0)=v_{0 y}$ the following conditions will be enough $\left(\beta=C_{0} / m\right)$

$$
\begin{gathered}
x=V_{0} t \cos \gamma_{0}-\left(V_{0} \cos \gamma_{0}-v_{0 x}\right)\left(1-\mathrm{e}^{-\beta t}\right) / \beta \\
y=\left(V_{0} \sin \gamma_{0}-g / \beta\right) t-\left(V_{0} \sin \gamma_{0}-v_{0 y}-g / \beta\right)\left(1-\mathrm{e}^{-\beta t}\right) / \beta
\end{gathered}
$$

The equations gave for $x(t)$ and $y(t)$ parametric form of pieces trajectory.

\section{Results:}

The rate of 2.2 pieces of the picture and the value $V_{0}$ of the parameter $\beta$ in different trajectory (Figure 2).

Issue 2:

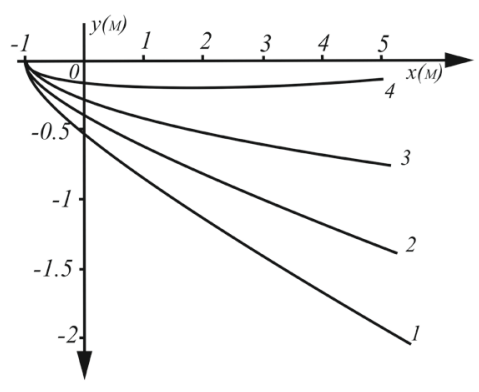

(a)

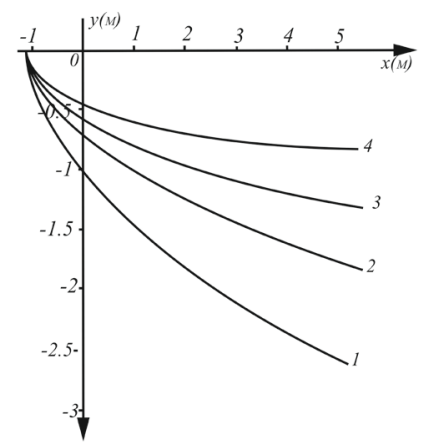

(c)

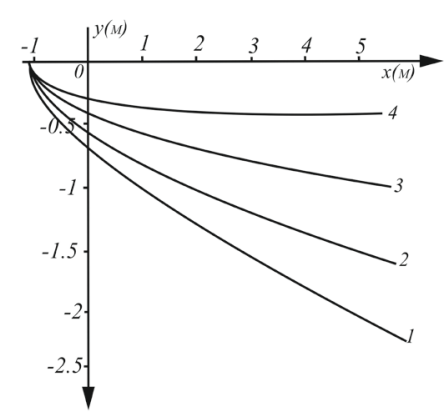

(b)

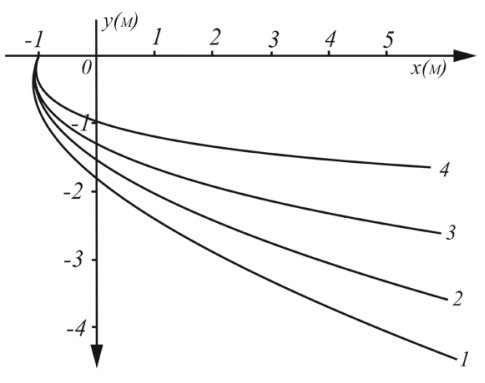

(d)

Figure 2. Speed air-influenced mainstream piece of pipe initial velocity [5]. $v_{0 y}=1 \mathrm{~m} / \mathrm{c}$ (a), $v_{0 y}=2 \mathrm{~m} / \mathrm{c}$ (b) and $v_{0 y}=5 \mathrm{~m} / \mathrm{c}, v_{0 y}=3 \mathrm{~m} / \mathrm{c}$ (c) and (d) the value of the parameter $\beta$ in various trajectories: $1-\beta=1,2-\beta=1.2,3-\beta=1.5,4-\beta=2$. 
Suppose surface of the rectangular $-2 b<x<0,-h<z<h$ tube of the flow cell transfer. Air particles $z=0(x 0 y)$ let it try the plain. Coordinate place $O$ and read from left to right starting point $0 x$ and $0 y$ cheers it up perpendicular oriented. Streaming pieces of cotton in the same plane is motioning $x=-b$ under the influence of a cyclical nature. Action point symmetric plane of movement $x>-b, y<0$, because we learn [6] [7].

For example, annexed weather can affect the trajectory $A B C D E$ of the flow of this (Figure 3)

$$
\begin{gathered}
y=y_{1}=k\left(x+x_{0}\right)\left[\frac{x+x_{0}}{2\left(x_{1}+x_{0}\right)}-1\right],-x_{0} \leq x \leq x_{1} \\
y=y_{2}(x)=-\frac{k\left(x_{1}+x_{0}\right)}{2}=\text { const }, x_{1} \leq x \leq x_{2} \\
y=y_{3}(x)=c+\left[y_{2}(x)-c\right] \frac{\left(x+x_{2}\right)^{2}}{\left(x_{3}-x_{2}\right)^{2}}, \quad x_{2} \leq x \leq x_{3}
\end{gathered}
$$

Here: $x_{0}\left(0 \leq x_{0} \leq b\right), x_{1}, x_{2}, x_{3}$-given abscess of trajectory line, $k$ and $c$-fixed numbers. $N E$ - as the length of the incision, $c$-piping the air.

Suppose a piece of pixel rate projections $\dot{x}=0, \dot{y}=-v_{0}$ respectively, which blocks the flow of air, which affect the trajectory of the camera movement people. In addition, her pieces in the vertical direction of movement of additional aerodynamic resistance Zhukovsky force $\mu \dot{y}$ ( $\mu$-coefficient of aerodynamic resistance force) away. Slices of the affected zones $A B, B C, B D$ and speed $V_{A B}, V_{B C}, V_{C D}$ and $V_{D E}$ and air flow is influenced look at the equation

Air flow on the direction of $A B$

$$
m \ddot{x}=C_{0}\left[V_{A B} \frac{x_{1}+x_{0}}{\sqrt{\left(x_{1}+x_{0}\right)^{2}+k^{2}\left(x-x_{1}\right)^{2}}}-\dot{x}\right]\left(-x_{0} \leq x \leq x_{1}\right)
$$

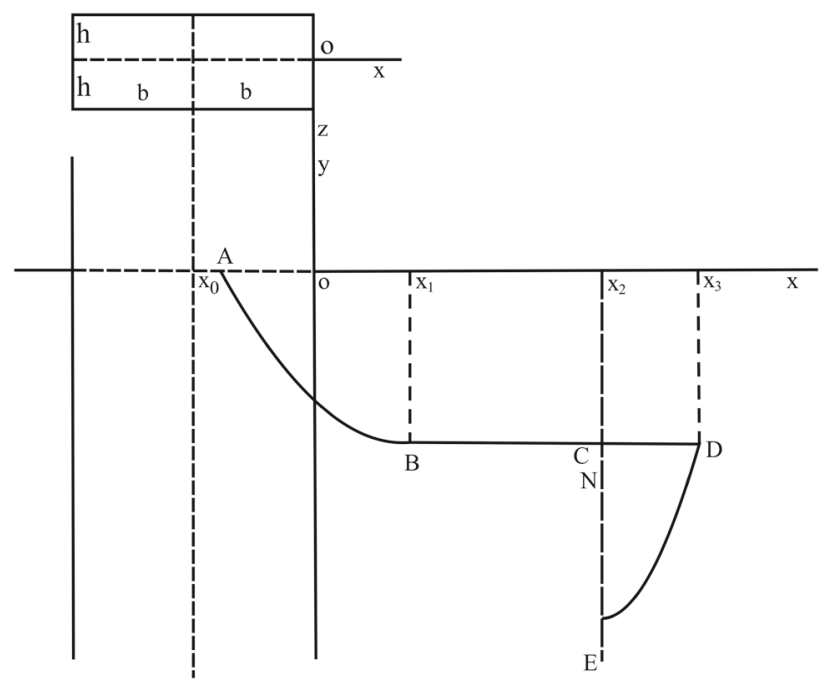

Figure 3. The flow of air movement trajectory scheme. 


$$
m \ddot{y}=C_{0}\left[V_{A B} \frac{k\left(x-x_{1}\right)}{\sqrt{\left(x_{1}+x_{0}\right)^{2}+k^{2}\left(x-x_{1}\right)^{2}}}-\dot{y}\right]-m g-\mu \dot{y}
$$

$B C\left(y=y_{2}(x)\right)$ air flow $\left(f^{\prime}=0\right)$

$$
\begin{gathered}
m \ddot{x}=C_{0}\left(V_{B C}-\dot{x}\right)\left(x_{1}<x<x_{2}\right) \\
m \dot{y}=-C_{0} \dot{y}-m g \\
m \ddot{x}=C_{0}\left(V_{C D}-\dot{x}\right)\left(x_{2}<x<x_{3}\right) \\
m \ddot{y}=-C_{0} \dot{y}-m g
\end{gathered}
$$

$D E$-airflow

$$
\begin{gathered}
m \ddot{x}=C_{0}\left[V_{D E} \frac{\left(x_{3}-x_{2}\right)^{2}}{\sqrt{\left(x_{3}-x_{2}\right)^{4}+\left(y_{2}(x)-c\right)^{2}\left(x-x_{1}\right)^{2}}}-\dot{x}\right]\left(x_{2} \leq x \leq x_{3}\right) \\
m \ddot{y}=C_{0}\left[V_{D E} \frac{\left(y_{2}(x)-c\right)\left(x-x_{1}\right)}{\sqrt{\left(x_{3}-x_{2}\right)^{4}+\left(y_{2}(x)-c\right)^{2}\left(x-x_{1}\right)^{2}}}-\dot{y}\right]-m g-\mu \dot{y}
\end{gathered}
$$

(2.12)-(2.16) equations systems $x=-x_{0}, y=0, \dot{x}=0, \dot{y}=-v_{0}, t=0$ are integrally in the tasks. We'll make the system like this:

$$
\begin{gathered}
m \ddot{x}+C_{0} \dot{x}=C_{0} V / \sqrt{1+f^{\prime 2}} \\
m \ddot{y}+C_{0} \dot{y}=C_{0} V f^{\prime} / \sqrt{1+f^{\prime 2}-m g-\mu \dot{y}}
\end{gathered}
$$

Here, $f^{\prime}=k\left(x-x_{1}\right) /\left(x_{1}+x_{0}\right), \quad V=V_{A B}-x_{0} \leq x \leq x_{1}$ it would be, $f^{\prime}=0$, $V=V_{B C} \quad x_{1} \leq x \leq x_{2} \quad$ would be $f^{\prime}=0, V=V_{C D} \quad x_{1} \leq x \leq x_{2} \quad$ would be $f^{\prime}=\left(y_{2}(x)-c\right)\left(x-x_{2}\right) /\left(x_{3}-x_{2}\right)^{2}, V=V_{D E} \quad x_{2} \leq x \leq x_{3}$ would be following.

Figure 4 shows Air flow (a) and (b), the slice of cotton in the value of the various titrate trajectories:

\section{Results:}

Figure 4 shows the air flow in the three tyrants and the impact on the slice of the value of the two trajectories. In consider, coordinate, and tyrant than the width of the tube and shake.

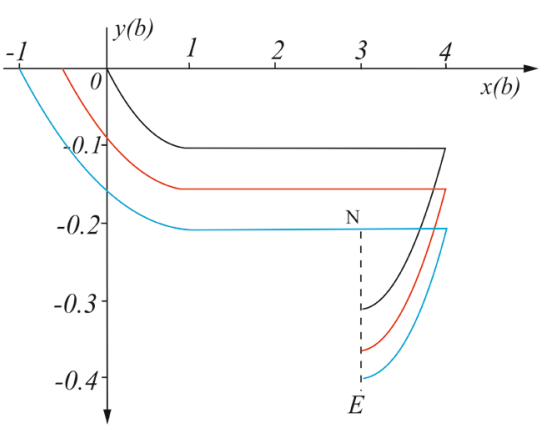

(a)

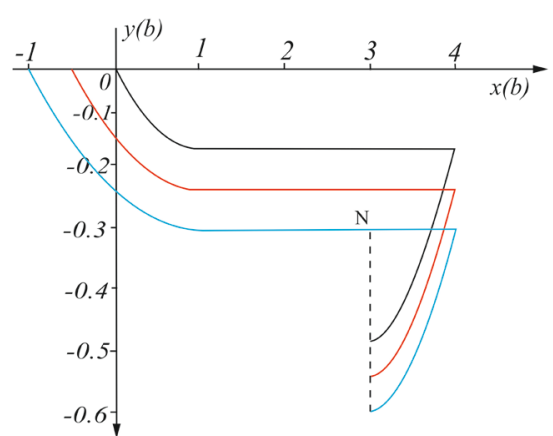

(b)

Figure 4. Airflow $c=0.2$ (a) and $c=0.4(\mathrm{~b})$, obsesses $x_{0}$ various value of the cotton slice effecting trajectories: $1-x_{0}=1,2-x_{0}=0.5,3-x_{0}=0$. 


\section{Conclusions}

The variability of the influence of the flow of the graphics analyses cells, as well as a variety of surfaces at the direction of the flow of air due to the absorption of an altered shape and influence trajectories in any coherent form remains the same value. It also exceeds the length of various restrictions on its surface; air is cooled with uneven distribution, and also far from initial abscesses to coordinate air in trajectories is limited to remote emergency sign.

An analysis of the vertical force of the weight of the pieces of cotton movement and the arrow on the impact of the additional resistance of the air force does not have great significance which was observed. In addition, the length of the restriction, it might sink more slices of cotton. For example, the primary abscesses barrier slices and cotton. If not, then set the starting abscesses only a slice of cotton, and keep the rest outside the obstacles.

\section{Conflicts of Interest}

The authors declare no conflicts of interest regarding the publication of this paper.

\section{References}

[1] Muradov, R. (2005) Ways to Improve the Efficiency of the Cotton Separator. Young Guard Tashkent.

[2] Amirov, R. (1975) The Movement of Raw Cotton per Separator Surface. Young Guard, Tashkent.

[3] Muradov, R. (2014) Cotton Basics of an Air Carrier to Increase the Effectiveness of the Device. Tashkent.

[4] Mamatqulov, O.T., Salohiddinova, M. and Muradov, R. (2017) Investigation of Separating Small Impurities and Heavy Compounds Using the Cotton Separator Equipment. American Journal of Science, Engineering and Technology, 2, 72-76. http://www.sciencepublishinggroup.com/j/ajset

[5] Nurmukhammadqizi, S.M., Muradovich, M.R., Tursunovich, M.O. and Sharipovich, K.S. (2020) Theoretical Research of the Process of Separating Impurities from Cotton Flow on the Vibrating Inclined Mesh Surface. International Journal of Advanced Science and Technology, 29, 10858-10869.

[6] Mukhamatxonovich, A.M., Makhamatjonovich, S.K., Muradovich, M.R. and Qizi, I.S.F. (2020) The Oretic Observation of the Cotton Movement in the Operating Camera of the New Separator. International Journal of Psychosocial Rehabilitation, 24, 6356-6364. https://www.psychosocial.com/article/PR2020619/27391/

[7] Makhamatjonovich, S.K., Makhkamov, A., Muradovich, M.R. and Karimov, A. (2020) Study of the Effect of the Mobile Floor of the Separator Device on the Cotton Section. International Journal of Psychosocial Rehabilitation, 24, 6473-6481. https://www.psychosocial.com/article/PR2020633/27917/ https://doi.org/10.37200/IJPR/V24I5/PR2020633 